\title{
Recent Advances in Near-Field to Far-Field Transformation Techniques
}

\author{
Claudio Gennarelli, ${ }^{1}$ Amedeo Capozzoli, ${ }^{2}$ Lars J. Foged, ${ }^{3}$ \\ Jeff Fordham, ${ }^{4}$ and Daniël Janse van Rensburg ${ }^{5}$ \\ ${ }^{1}$ Dipartimento di Ingegneria Elettronica ed Ingegneria Informatica, Università di Salerno, Via Ponte Don Melillo, \\ 84084 Fisciano, Italy \\ ${ }^{2}$ Dipartimento di Ingegneria Biomedica, Elettronica e delle Telecomunicazioni, Università di Napoli Federico II, Via Claudio 21, \\ 80125 Naples, Italy \\ ${ }^{3}$ SATIMO Italian Office, Via Castelli Romani 59, 00040 Pomezia, Italy \\ ${ }^{4}$ MI Technologies, Suite 100, 1125 Satellite Boulevard, Suwanee, GA 30024-4629, USA \\ ${ }^{5}$ Nearfield Systems Inc.19730 Magellan Drive, Torrance, CA 90503, USA
}

Correspondence should be addressed to Claudio Gennarelli, gennar@diiie.unisa.it

Received 28 May 2012; Accepted 28 May 2012

Copyright ( $) 2012$ Claudio Gennarelli et al. This is an open access article distributed under the Creative Commons Attribution License, which permits unrestricted use, distribution, and reproduction in any medium, provided the original work is properly cited.

For electrically large antenna systems, far-field (FF) range size limitations, transportation, and mounting problems make it difficult or absolutely impractical to measure the radiation pattern using conventional FF ranges. On the other hand, the increase of high-performance antennas, as those employed in radar and satellite systems, requires more accurate and complete measurements of their radiating characteristics, generally requiring the use of controlled indoor anechoic chambers, to overcome drawbacks associated with outdoor FF measurements. As a consequence, the problem of determining the antenna FF pattern from near-field (NF) measurements has attracted considerable attention in the last fifty years [1-11].

Indeed, NF scanning techniques have become the best choice as long as complete pattern and polarization measurements are required. In addition they allow to determine the field at the surface of the antenna, even if generally, within the "visible" resolution, which can be exploited usefully to determine surface errors in reflector antennas as well as to identify faulty elements in arrays.

Generally speaking, NF antenna characterization can exploit complex field data or phaseless field data, the first choice being the most commonly used in practice.

In an NF system processing complex field data, a probe antenna is moved along a surface (the scanning surface) whose shape is typically planar, cylindrical, or spherical. It collects complex voltage samples, which, together with their positions, allow the desired quantities to be computed. For instance, from the phase and amplitude data, and taking into account the probe effect, the FF pattern can be computed. Commonly, the measured NF data are transformed into FF patterns by using an expansion of the field radiated by the antenna under test (AUT) in terms of modes, that is, a complete set of solutions of the vector wave equation in the region exterior to the antenna. Plane, cylindrical, or spherical waves are generally used. The type of modal expansions used for representing the field is typically determined by the shape of scanning surface, which, accordingly, will be a plane, a cylinder, or a sphere, respectively. The orthogonality properties of the modes on such surfaces are then exploited to calculate the modal expansion coefficients, allowing the reconstruction of the AUT far field.

The use of different scanning geometries is justified from the fact that each approach has its own specific advantages, depending on both the AUT characteristics and the measurement requirements.

The aim of this special issue is to provide an international forum for the researchers working in the antenna measurement field to disseminate new ideas and describe recent advances on NF methods in characterization techniques, simulations, and applications. 
This special issue collects ten papers from 29 authors, belonging to several countries and coming from universities, research institutions, and companies. These papers cover many of the hot topics related to NF-FF transformation techniques.

The topic "mitigation of the error due to the measurement area truncation" is addressed in the paper "Reduction of truncation errors in planar, cylindrical, and partial spherical near-field antenna measurements" by F. J. Cano-Fácila et al. and in the other "Using truncated data sets in spherical scanning antenna measurements" by R. C. Wittmann et al.

The paper "Numerical investigation of the systemmatrix method for higher-order probe correction in spherical near-field antenna measurements" by T. B. Hansen and that "Application of nonredundant sampling representations of electromagnetic fields to NF-FF transformation techniques" by O. M. Bucci and C. Gennarelli fall within the topic "advances in NF-FF transformation techniques."

The theme "NF probe design and characterization" is covered in the paper "Near-field antenna measurements using photonic sensor of Mach-Zehnder interferometer" by M. Hirose et al.

The paper "A microwave holographic procedure for large symmetric reflector antennas using a Fresnel-zone field data processing" by G. Mazzarella, G. Montisci, and G. Serra deals with the topic "microwave holography."

Innovative NF measurement techniques is the theme of the paper "A probe-compensated helicoidal NF-FF transformation for aperture antennas using a prolate spheroidal expansion" by A. Capozzoli et al. as well as of "An innovative direct NF-FF transformation technique with helicoidal scanning" by F. D’Agostino et al.

The topic "electromagnetic algorithms and data processing" is dealt with in the paper "Examination of farfield mathematical absorber reflection suppression through computational electromagnetic simulation" by S. Gregson et al. and in the other "Development of a near-field bistatic synthetic aperture radar for complex target reconstruction" by D. G. Johnson and G. M. Brooker.

A brief description of each of the papers contained in this special issue is reported in the following for the reader's convenience, being the presentation order just related to the publication date order.

In the paper by F. J. Cano-Fácila et al., they present an effective method to reduce the truncation error when measuring the field in the most common truncated scanning surfaces (plane, cylinder, and partial sphere). The method makes use of the classical Gerchberg-Papoulis algorithm, widely used in the literature to extrapolate band-limited signals.

The paper by T. B. Hansen concerns a detailed numerical investigation of the system-matrix method, recently proposed by the same author, for higher-order probe correction in the NF-FF transformation with spherical scanning.

The paper by O. M. Bucci and C. Gennarelli presents an overview of the application of the nonredundant sampling representations of electromagnetic (EM) fields to NFFF transformations with conventional or spiral scanning, outlining the remarkable reduction in the number of needed NF samples and measurement time so achievable.

A new holographic procedure for the diagnosis of large reflector antennas is proposed in the paper by G. Mazzarella et al. The procedure is based on the direct use of Fresnel-field data and employs a regularized singular value decomposition technique.

The paper by M. Hirose et al. deals with a photonic sensor of the Mach-Zehnder interferometer type allowing the electric field measurement in the very near-field range of the AUT. It is shown that such a sensor can be applied to planar, spherical, and cylindrical NF measurements without any probe compensation approximately below $10 \mathrm{GHz}$.

A probe-compensated NF-FF transformation with helicoidal scanning for aperture antennas is presented in the paper by A. Capozzoli et al. It exploits a proper aperture field expansion, based on the use of the prolate spheroidal wave functions, accounting for the a priori information on the AUT and allows a significant reduction of the field data.

In the paper by R. C. Wittmann et al., they propose a method to mitigate the errors arising in the spherical scanning measurements when the data are not collected over an entire sphere. The technique uses a least-square estimation method with an energy constraint.

A direct NF-FF transformation with helicoidal scanning, which allows the evaluation of the antenna far field from a minimum set of NF data without interpolating them, is developed in the paper by F. D'Agostino et al. It relies on the nonredundant sampling representation of EM fields and matches the advantage of the fast helicoidal scanning with those of the direct cylindrical NF-FF transformation.

The paper by S. F. Gregson et al. concerns a new approach for suppressing the effect of spurious scattering in the far field as an extension of techniques already presented for NF measurements. A numerical simulator able to evaluate the antenna field in the presence of scattering objects is employed to investigate the proposed technique.

In the paper by D. G. Johnson and G. M. Brooker, they illustrate the development of a near-field bistatic inverse synthetic aperture radar for the analysis of rock samples. Moreover, they discuss an imaging algorithm matched to spherical shapes by providing numerical and experimental inversions.

\section{Acknowledgments}

We wish to thank all authors, who have decided to present some relevant aspects of their research activities in such a special issue, and the reviewers, whose suggestions have contributed to improve the quality of the papers. Finally, a very special acknowledgement is due to the Editorial Board of the International Journal of Antennas and Propagation who has made possible the publication of this special issue.

Claudio Gennarelli Amedeo Capozzoli

Lars J. Foged Jeff Fordham

Daniël Janse van Rensburg 


\section{References}

[1] R. C. Johnson, H. A. Ecker, and J. S. Hollis, "Determination of far-field antenna patterns from near-field measurements," Proceedings of the IEEE, vol. 61, no. 12, pp. 1668-1694, 1973.

[2] J. Appel-Hansen, J. D. Dyson, E. S. Gillespie, and T. G. Hickman, "Antenna measurements," in The Handbook of Antenna Design, A. W. Rudge, K. Milne, A. D. Olver, and P. Knight, Eds., chapter 8, Peter Peregrinus Ltd, London, UK, 1982.

[3] A. D. Yaghjian, "An overview of near-field antenna measurements," IEEE Transactions on Antennas and Propagation, vol. AP-34, no. 1, pp. 30-45, 1986.

[4] E. S. Gillespie, Ed., "Special Issue on near-field scanning techniques," IEEE Transactions on Antennas and Propagation, vol. AP-36, no. 6, pp. 727-901, 1988.

[5] J. Hald, J. E. Hansen, F. Jensen, and F. H. Larsen, Spherical Near-Field Antenna Measurements, Peter Peregrinus Ltd, London, UK, 1988.

[6] G. E. Evans, Antenna Measurement Techniques, , Artech House, Boston, Mass, USA, 1990.

[7] D. Slater, Near-Field Antenna Measurements, Artech House, Boston, Mass, USA, 1991.

[8] C. Gennarelli, G. Riccio, F. D’Agostino, F. Ferrara, and R. Guerriero, Near-Field_Far-Field Transformation Techniques, vol. 1, CUES, Salerno, Italy, 2004.

[9] C. Gennarelli, G. Riccio, F. D’Agostino, F. Ferrara, and R. Guerriero, Near-Field_Far-Field Transformation Techniques, vol. 2, CUES, Salerno, Italy, 2006.

[10] S. F. Gregson, J. McCormick, and C. G. Parini, Principles of Planar Near-Field Antenna Measurements, The Institution of Engineering and Technology, London, UK, 2007.

[11] M. H. Francis and R. W. Wittmann, "Near-field scanning measurements: theory and practice," in Modern Antenna Handbook, C. A. Balanis, Ed., chapter 19, John Wiley \& Sons, Hoboken, NJ, USA, 2008. 

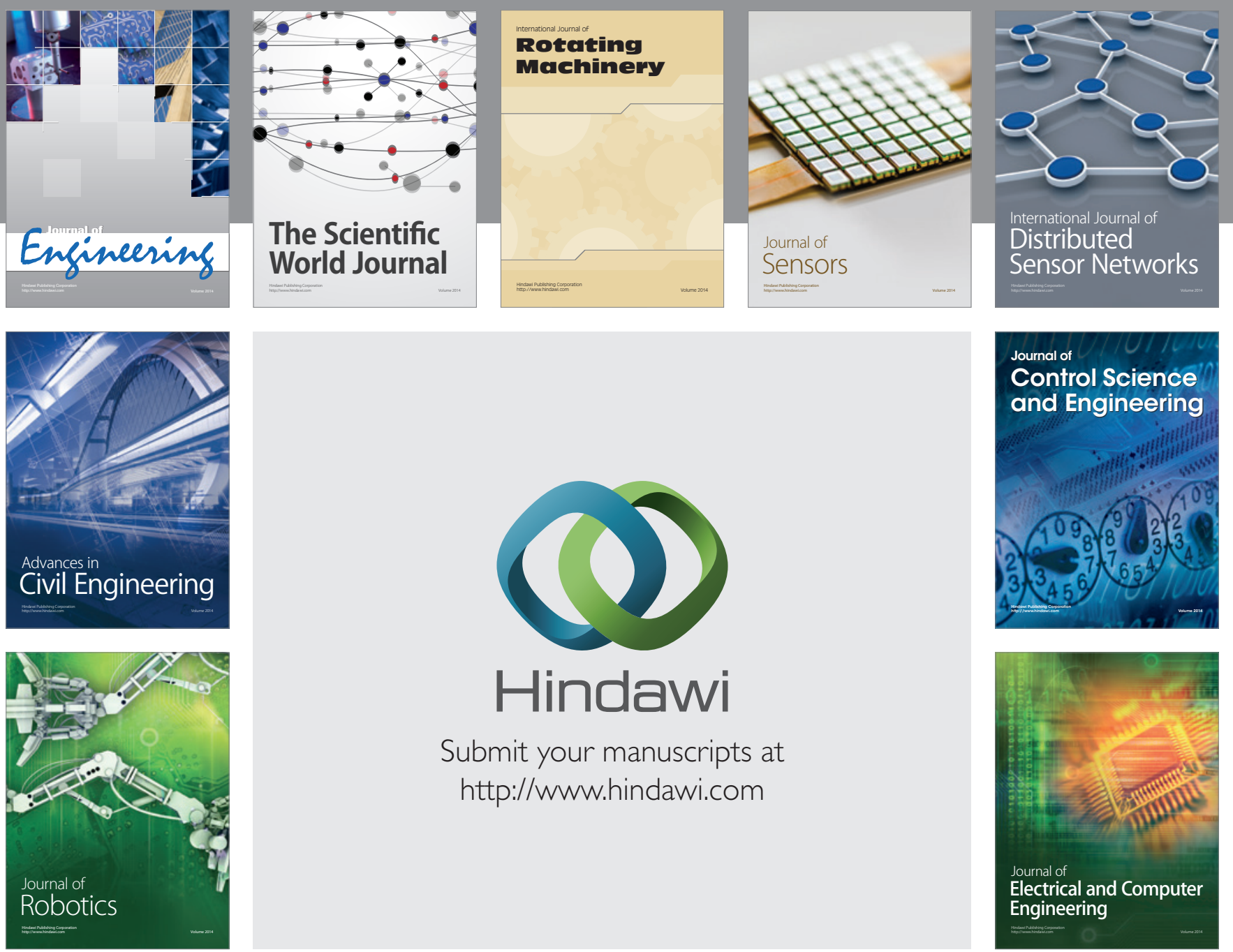

Submit your manuscripts at

http://www.hindawi.com
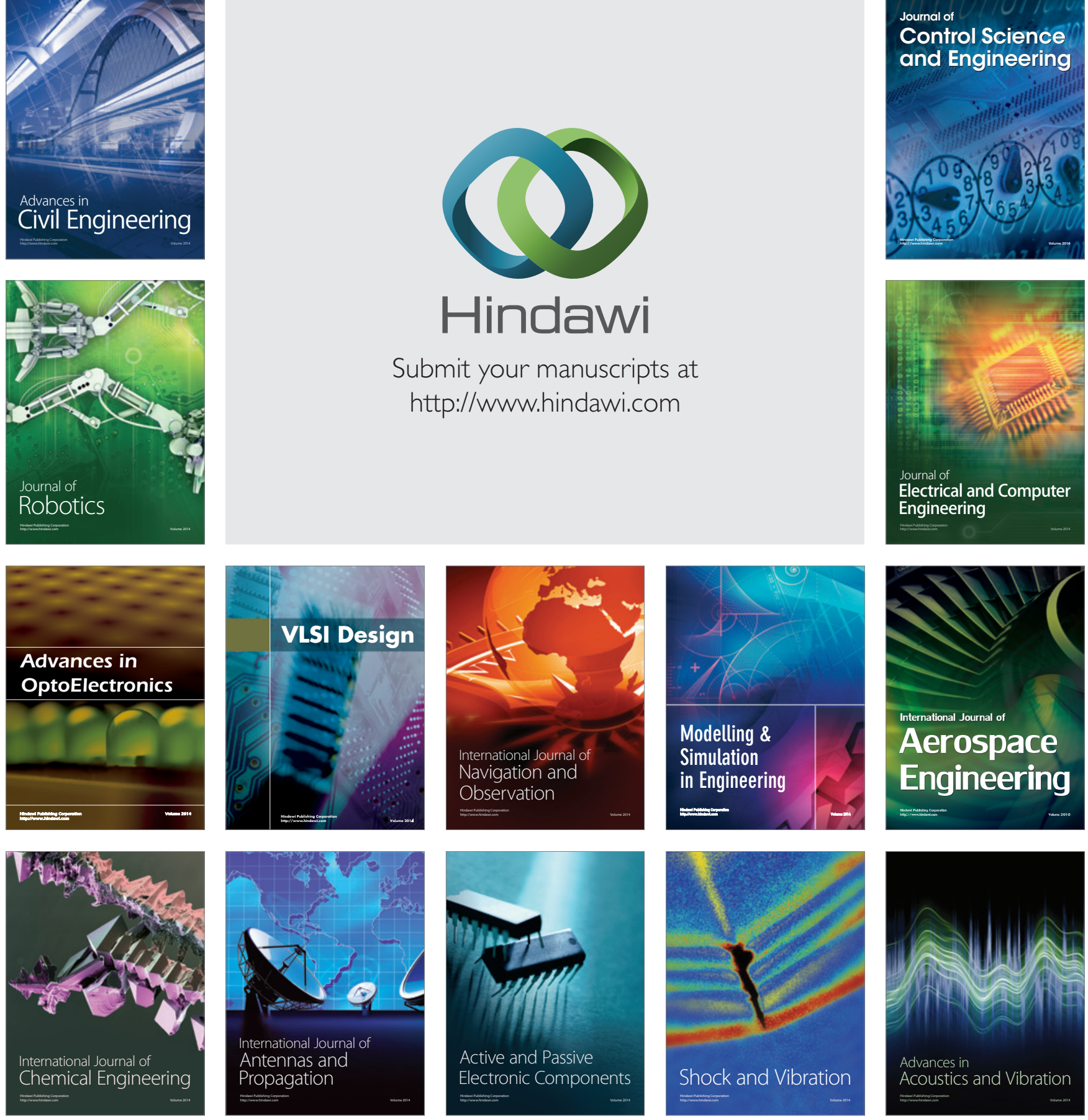